\title{
Visible light photodegradation of dyes and paracetamol by direct sensitization mechanism onto metallic $\mathrm{MoO}_{2}$ nanocrystals
}

\author{
Alessandro Di Mauro ${ }^{a}$, Marta Maria Natile ${ }^{b}$, Anton Landström ${ }^{c}$, Isabella Concina ${ }^{c}$, \\ Matteo Ferroni ${ }^{\mathrm{d}, \mathrm{e}}$, Vittorio Privitera ${ }^{\mathrm{a}}$, Giuliana Impellizzeri ${ }^{\mathrm{a}}$, Mauro Epifani ${ }^{\mathrm{f}, *}$ \\ ${ }^{a}$ CNR-IMM, Via S. Sofia 64, 95123 Catania, Italy \\ ${ }^{\mathrm{b}}$ Istituto di Chimica della Materia Condensata e Tecnologie per l'Energia, Consiglio Nazionale delle Ricerche (ICMATE-CNR) and Dipartimento di Scienze Chimiche, \\ Università di Padova, Via F. Marzolo 1, 35131 Padova, Italy \\ ${ }^{\mathrm{c}}$ Luleå University of Technology, 97187 Luleå, Sweden \\ d Department of Information Engineering, University of Brescia, Via Branze, Brescia, Italy \\ ${ }^{\text {e }}$ CNR-IMM, Via Gobetti, Bologna, Italy \\ ${ }^{\mathrm{f}}$ CNR-IMM, Via Monteroni c/o Campus Universitario, 73100 Lecce, Italy
}

\section{A R T I C L E I N F O}

\section{Keywords:}

Photodegradation

Dye sensitization

Direct sensitization mechanism

Methylene blue

$\mathrm{MoO}_{2}$

Solvothermal synthesis

\begin{abstract}
A B S T R A C T
$\mathrm{MoO}_{2}$ nanocrystals were prepared by solvothermal treatment of a Mo chloromethoxide at $250{ }^{\circ} \mathrm{C}$ in oleic acid. The monoclinic $\mathrm{MoO}_{2}$ phase, with a mean crystallite size of $29 \mathrm{~nm}$, formed through reduction of molybdenum bronzes. The as-prepared $\mathrm{MoO}_{2}$ nanocrystals were free from organics, allowing their use in photodegradation tests of organic pollutants (methylene blue, rhodamine B, paracetamol), without any preliminary purification treatment of the nanocrystals. It was found that $\mathrm{MoO}_{2}$ was an efficient adsorbent of methylene blue $\left(43 \mathrm{mg} \mathrm{g}^{-1}\right.$ for $1.5 \times 10^{-4} \mathrm{M}$ concentration) in the dark and an efficient photodegradation catalyst under visible light (all methylene blue removed from the solution after $240 \mathrm{~min}$ ). From the analysis of the combined photodegradation tests of rhodamine B and paracetamol, it was clarified that direct sensitization was responsible for photodegradation. This finding was related to the work function value of metallic $\mathrm{MoO}_{2}$, placed at more negative values if compared with other metallic materials.
\end{abstract}

\section{Introduction}

Molybdenum dioxide $\left(\mathrm{MoO}_{2}\right)$ crystallizes in its most stable form in the monoclinic crystallographic system [1] and features an interesting metallic electrical conductivity. It has long been known as a catalyst but it has triggered renewed interest due to its applications in batteries [2, 3], supercapacitors [4,5], water splitting [6-9] and SERS spectroscopy [10]. Very recently, $\mathrm{MoO}_{2}$ has showed very interesting photodegradation properties towards several common water pollutants. [4, 11]. Such a property is quite unexpected with respect to the electronic properties of $\mathrm{MoO}_{2}$. The latter have been investigated for long time as a part of the general effort to understand the physical properties of transition metal oxides. Briefly, $\mathrm{MoO}_{2}$ belongs to a class of oxides where the intercation distance is such to allow electronic coupling of neighbouring Mo cations, which is manifested by splitting of their $d$ orbitals. The orbitals oriented along the $c$ axis are split into two largely separated bands $\left(\mathrm{t}||\right.$ and $\mathrm{t} \mid \|^{*}$ ), among which the antibonding $\pi^{*}$ band (derived from O-Mo bonding) is located. Of the two $d$ electrons of Mo, one will fill the $\mathrm{t} \|$ band and the other will partially fill the $\pi^{*}$ band. Being the Fermi level located into the $\pi *$ band, the material displays metallic behavior. This view was early proposed by Goodenough [12-14] and Rogers et al. [15] and further refined by cluster and tight binding calculations by Sasaki et al. [16] and Burdett [17]. Later on, full band structure DFT calculation was carried out $[18,19]$, strongly substantiating the early models. Based on the picture by Goodenough, the optical absorption increase above $2.5 \mathrm{eV}$ in $\mathrm{MoO}_{2}$ was mainly ascribed to excitation from the top of the oxygen $p$ band to the Fermi level in the $4 d$ bands [20,21]. In Ref. [11] a cutoff filter at $420 \mathrm{~nm}$ was used for the photocatalytic studies. Therefore, if a standard view of the photocatalytic effect is assumed, based on photogenerated electron-hole couples in a semiconductor, a very narrow region of visible light would be available for photodegradation. In the present work a peculiar synthesis of $\mathrm{MoO}_{2}$ nanoparticles is presented, capable of providing organics-free material without any purification. This property allowed planning the use of

\footnotetext{
* Corresponding author.

E-mail address: maurosalvatore.epifani@cnr.it (M. Epifani).
} 
$\mathrm{MoO}_{2}$ in photocatalytic tests, since no pre-processing of the materials was necessary. It was found that $\mathrm{MoO}_{2}$ is a powerful photocatalyst for the degradation of methylene blue (MB), working under the visible light of a solar simulator. Moreover, it was shown for the first time that $\mathrm{MoO}_{2}$ is an efficient adsorbent of MB in the dark, so paving the way to the combined use of adsorption/photodegradation for the elimination of organic aqueous contaminants. More importantly, the present work stresses the interpretation of the photodegradation results in terms of the correct view of the band structure of $\mathrm{MoO}_{2}$, by properly classifying it as a metal.

\section{Experimental}

\subsection{Synthesis of $\mathrm{MoO}_{2}$ nanocrystals}

For preparing $\mathrm{MoO}_{2}$ nanoparticles, $2 \mathrm{~mL}$ of Mo chloromethoxide, prepared as previously described [22], were dispersed in $10 \mathrm{~mL}$ of oleic acid (90\%, technical grade). The resulting opaque suspension was poured into a glass vial, which was inserted into a $45 \mathrm{~mL}$ autoclave, heated into an oven at a rate of $5^{\circ} \mathrm{C} / \mathrm{min}$ up to temperatures ranging from 175 to $250^{\circ} \mathrm{C}$, and kept at such temperatures for $2 \mathrm{~h}$. After cooling, the product was recovered by addition of methanol and centrifugation, washed in acetone and finally dried at $90^{\circ} \mathrm{C}$. Dark blue powders were obtained, very fine and free flowing.

\subsection{Materials characterization}

X-ray diffraction (XRD) patterns were recorded on a PANalytical Empyrean diffractometer. $\mathrm{Cu} \mathrm{K}-\alpha$ radiation was obtained with a $\mathrm{Cu}$ anode operated at $45 \mathrm{kV} / 40 \mathrm{~mA}$ and a graphite monochromator. A programmable divergence slit was used to illuminate a $10 \times 10 \mathrm{~mm}^{2}$ area of the sample surface. Rietveld refinement of the $\mathrm{MoO}_{2}$ pattern was carried out with the MAUD software [23].

XPS spectra were taken with a Perkin Elmer PHI 5600 spectrometer. Both extended spectra (survey: $187.85 \mathrm{eV}$ pass energy, $0.5 \mathrm{eV}$ step, $0.025 \mathrm{~s} \cdot \mathrm{step}^{-1}$ ) and detailed spectra (Mo $3 \mathrm{~d}$, O $1 \mathrm{~s}$ and $\mathrm{C} 1 \mathrm{~s}: 23.5 \mathrm{eV}$ pass energy, $0.1 \mathrm{eV}$ step, $0.2 \mathrm{~s} \cdot \mathrm{step}^{-1}$ ) were collected with a standard $\mathrm{Al}-\mathrm{K} \alpha$ source $(1486.6 \mathrm{eV})$ working at $250 \mathrm{~W}$.

Fourier Transform Infrared (FTIR) measurements were carried out by a Nicolet 6700 spectrometer in diffuse reflectance setup, after dispersing the sample powders in $\mathrm{KBr}$.

The specific surface area and porosity of the nanostructured samples were measured by the Brunauer-Emmett-Teller (BET) method using $\mathrm{N}_{2}$ adsorption/desorption at $77 \mathrm{~K}$ with a Quantachrome NOVA 2200e series surface analyzer.

\subsection{Adsorption and photodegradation experiments}

The adsorption properties of $\mathrm{MoO}_{2}$ nanocrystals were evaluated through the adsorption of $\mathrm{MB}$ dye in aqueous solution. In a typical experiment, $1 \mathrm{mg}$ of $\mathrm{MoO}_{2}$ sample was added to $2 \mathrm{~mL}$ of MB solution with a starting concentration of $1.5 \times 10^{-4} \mathrm{M}$. The test was run in parallel for both materials. At regular time intervals, the solutions were collected and centrifuged at $4000 \mathrm{rpm}$ for $5 \mathrm{~min}$, so to separate the nanopowders. The variation of the MB concentration, during the adsorption experiment, was evaluated spectrophotometrically (using a PerkinElmer Lambda 45 UV-vis spectrophotometer) via the solution absorbance at $664 \mathrm{~nm}$. In order to use the Lambert-Beer law, the solution was diluted before the analysis to have the value of absorbance of $\sim 1$ [24]. The dye adsorption on the beaker walls was also checked, as a reference, in the absence of $\mathrm{MoO}_{2}$. The photocatalytic activity of $\mathrm{MoO}_{2}$ under visible light was evaluated by following the degradation of $\mathrm{MB}$ in aqueous solution, with the same protocol described before, by using an Oriel VeraSol TM Solar Simulator (Newport) with an irradiance of $12 \mathrm{~mW} / \mathrm{cm}^{2}$. Solutions of Rhodamine $\mathrm{B}(\mathrm{RhB})$ and paracetamol were also tested to investigate the dye sensitization mechanism. The RhB test was first carried out using

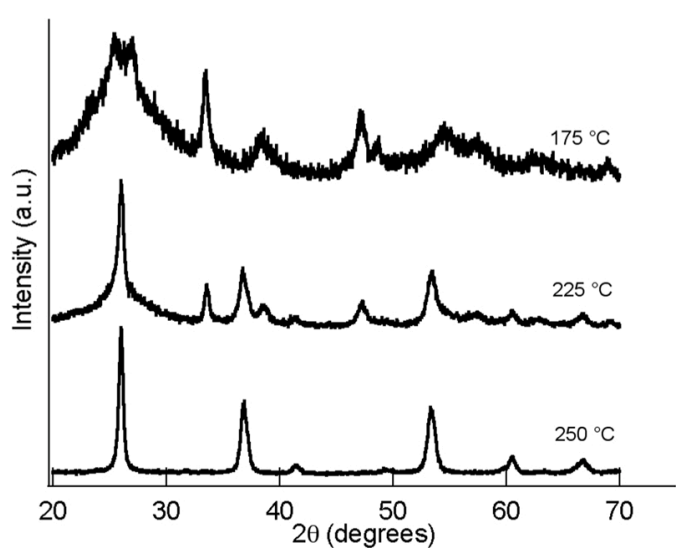

Fig. 1. XRD patterns of the samples obtained by solvothermal treatment at the indicated temperatures. All the reflections in the $250{ }^{\circ} \mathrm{C}$ pattern belong to the $\mathrm{MoO}_{2}$ monoclinic phase.

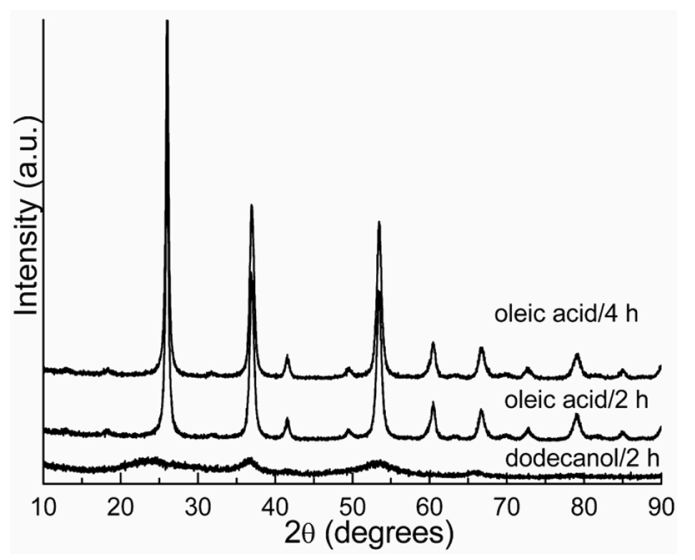

Fig. 2. XRD patterns of the samples obtained by solvothermal treatment in the indicated conditions. The patterns have been vertically shifted for clarity.

the full solar spectrum and then repeated by filtering off the $500-600 \mathrm{~nm}$ range which contains the absorbance band of RhB. The measurements were performed with the same procedures described above for the MB photodegradation. The starting solution included paracetamol and RhB with a concentration of $1.5 \times 10^{-5} \mathrm{M}$ and $9.5 \times 10^{-5}$ in deionized water, in order to have for both compounds an absorbance of about 1 . The degradation of paracetamol and of RhB was evaluated by following the absorbance peak at $243 \mathrm{~nm}$ and $546 \mathrm{~nm}$, respectively, in the Lambert-Beer regime.

\section{Results and discussion}

\subsection{Materials synthesis and growth pathways}

Fig. 1 reports the XRD patterns of samples obtained by heattreatment for $2 \mathrm{~h}$ at different temperatures. It is clear that the final $\mathrm{MoO}_{2}$ phase developed through other crystalline phases which were present already after treatment at $175^{\circ} \mathrm{C}$. Monoclinic $\mathrm{MoO}_{2}$ (tugarinovite), in particular, was observed after treatment at $225^{\circ} \mathrm{C}$, but it was accompanied by residuals of the phase present at $175^{\circ} \mathrm{C}$, and conversion was complete only by processing at $250^{\circ} \mathrm{C}$.

Inspection of the $175^{\circ} \mathrm{C}$ pattern revealed that the related phase was most probably a molybdenum bronze, as suggested by comparison with the patterns published in the past $[25,26]$. The phase could actually be a mixture of $\mathrm{H}_{0.34} \mathrm{MoO}_{3}$ (type I) and $\mathrm{H}_{0.93} \mathrm{MoO}_{3}$ (type II) bronzes, given the similarity of their XRD reflections. This hypothesis was confirmed by the FTIR analysis shown in Fig. 5. In the following, the mixture of phases 


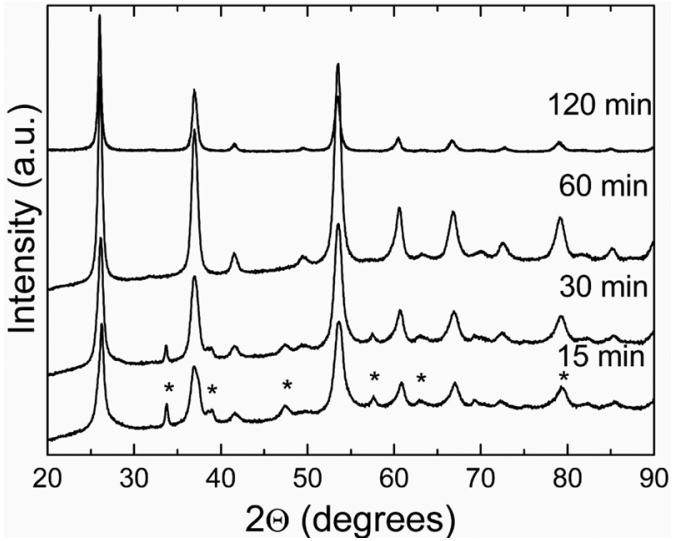

Fig. 3. XRD patterns of the samples obtained by solvothermal treatment at $250^{\circ} \mathrm{C}$ for the indicated times. The stars indicate the reflections of the molybdenum bronze phase(s).

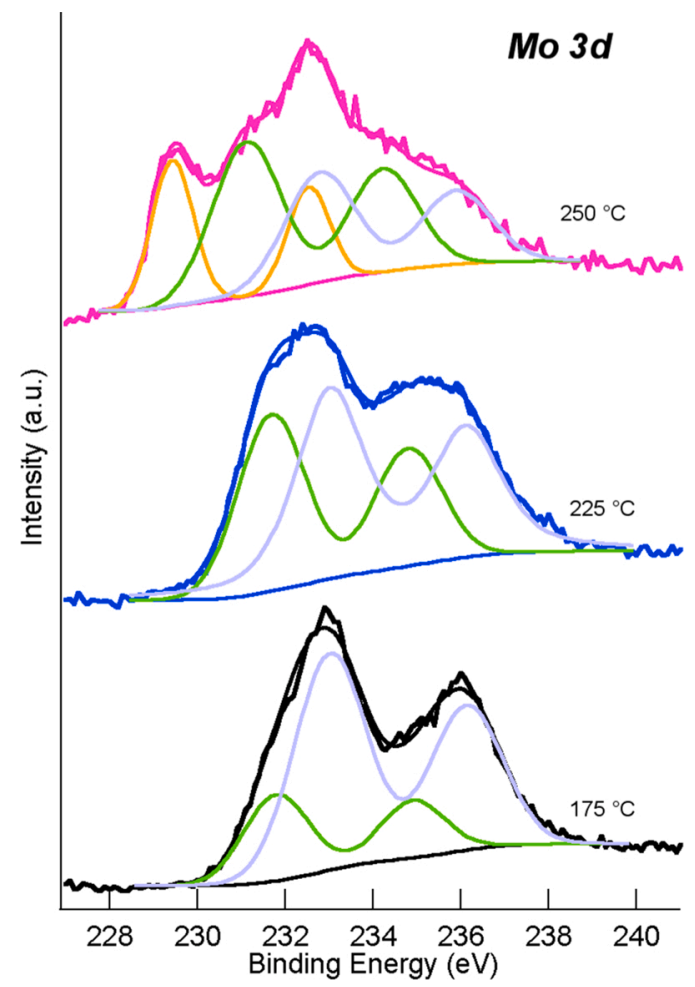

Fig. 4. Mo 3d XPS peaks of the samples prepared at the indicated temperatures. The doublets characteristic of molybdenum in different oxidation states are reported in different colors: Mo(IV) - orange, Mo(V) - green and Mo(VI) - gray.

will be referred to as MOB. The synthesis conditions were further explored by changing the solvent and the heating time. Dodecanol was chosen since in the case of $\mathrm{WO}_{3}$ it allowed preparing more unusual phase and morphology [27]. The XRD results are shown in Fig. 2, and it can be seen that oleic acid was a necessary choice to establish the $\mathrm{MoO}_{2}$ phase. Dodecanol resulted in a pattern whose weak reflections seemed related to those of $\mathrm{MoO}_{2}$, despite largely shifted, so it was not further considered. Moreover, heating at $250^{\circ} \mathrm{C}$ in oleic acid for both 2 and $4 \mathrm{~h}$ resulted in a mean $\mathrm{MoO}_{2}$ domain size of $29 \mathrm{~nm}$, as obtained by Rietveld refinement of the patterns (see Supporting Information) so $2 \mathrm{~h}$ was kept as a standard heating time in all the experiments.

For gaining more insight into the precursor evolution, another set of experiments was designed, where isothermal heating was carried out at $250{ }^{\circ} \mathrm{C}$ for several time intervals.

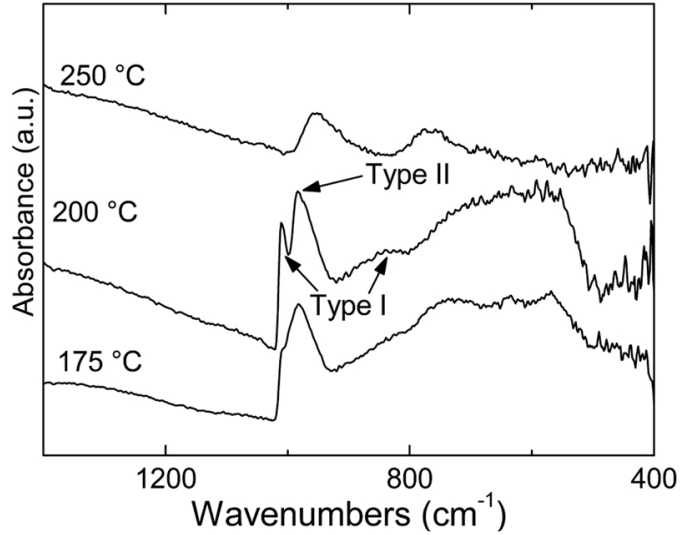

Fig. 5. FTIR spectra of the samples obtained by solvothermal treatment at the indicated temperatures.

Table 1

Mo 3d XPS Peak Positions (Binding Energy, eV) of the samples in Fig. 4.

\begin{tabular}{|c|c|c|c|c|c|c|}
\hline & \multicolumn{2}{|c|}{ Mo(IV) } & \multicolumn{2}{|l|}{$\mathrm{Mo}(\mathrm{V})$} & \multicolumn{2}{|c|}{ Mo(VI) } \\
\hline & $3 d_{5 / 2}$ & $3 d_{3 / 2}$ & $3 d_{5 / 2}$ & $3 d_{3 / 2}$ & $3 d_{5 / 2}$ & $3 d_{3 / 2}$ \\
\hline $175^{\circ} \mathrm{C}$ & - & & 231.8 & 234.9 & 233.0 & 236.1 \\
\hline $225^{\circ} \mathrm{C}$ & - & & 231.7 & 234.8 & 233.0 & 236.1 \\
\hline $250^{\circ} \mathrm{C}$ & 229.4 & 232.5 & 231.1 & 234.3 & 232.8 & 235.9 \\
\hline
\end{tabular}

The related XRD patterns are presented in Fig. 3. It can be seen that, after $15 \mathrm{~min}$, the $\mathrm{MoO}_{2}$ structure was developed but it was overlapped with the MOB phase (same reflections of Fig. 1). The MOB phase progressively decreased for longer heating times and disappeared after $2 \mathrm{~h}$ at $250^{\circ} \mathrm{C}$. After $1 \mathrm{~h}$ it seems that a residual reflection is present at $2 \theta \sim 63^{\circ}$. Hence the MOB phases appeared stable for at least $2 \mathrm{~h}$ at $175^{\circ} \mathrm{C}$ (Fig. 1). Moreover, they were also partially preserved at $250^{\circ} \mathrm{C}$, as seen in Fig. 3, for kinetic reasons (the conversion to $\mathrm{MoO}_{2}$ is not so fast to have the MOB immediately consumed as soon as the autoclave gets to $250^{\circ} \mathrm{C}$ ). Such MOB phase stability allowed hypothesizing that the precursor evolution started directly from the MOB formation, which then evolved to the finally stable $\mathrm{MoO}_{2}$. The exact formation temperature of MOB of course cannot be predicted from the data of Fig. 1. It can also be observed that the $\mathrm{MoO}_{2}$ reflections in Fig. 3 shifted to lower angles as the heating time increased. This effect was particularly evident for the envelope at $2 \theta \sim 27^{\circ}$. Such shift indicated an increase of the related crystal planes distance in the $\mathrm{MoO}_{2}$ lattice. $\mathrm{MoO}_{2}$ formation by condensation of neighboring $\mathrm{Mo}-\mathrm{OH}$ bonds would result in lattice contraction. Therefore the $\mathrm{MoO}_{2}$ formation from the MOB phase must involve complex reconstruction of the lattice upon loss of $\mathrm{OH}$ groups, favored by oleic acid. The direct formation of MOB phases was surprising since they have never been directly synthesized without reduction of Mo(VI) compounds, to the best of our knowledge.

The XPS spectra of the same sequency of samples of Fig. 1 were then acquired. Fig. 4 shows the XPS spectra of the Mo 3d core level. Only Mo (V) and Mo (VI) were detected in the samples prepared at lower temperature $\left(175\right.$ and $\left.225^{\circ} \mathrm{C}\right)$. A thermal treatment at $250^{\circ} \mathrm{C}$ was necessary to observe also a contribution of Mo (IV) (Fig. 4 and Table 1). In this sample three different oxidation states of Mo were detected. These different oxidation states clearly reinforced the view that a molybdenum bronze constituted the $175^{\circ} \mathrm{C}$ phase, so resulting in a mixture of oxidation states higher than Mo (IV). Further reduction in the oleic acid environment resulted in $\mathrm{MoO}_{2}$.

FTIR spectroscopy was used as a final tool for elucidating the nature of the MOB phase. The spectra sequency in Fig. 5 clearly shows, following previous works $[25,28]$, that the MOB phase is actually a mixture of the type I and type II molybdenum bronzes. Expectedly, with 

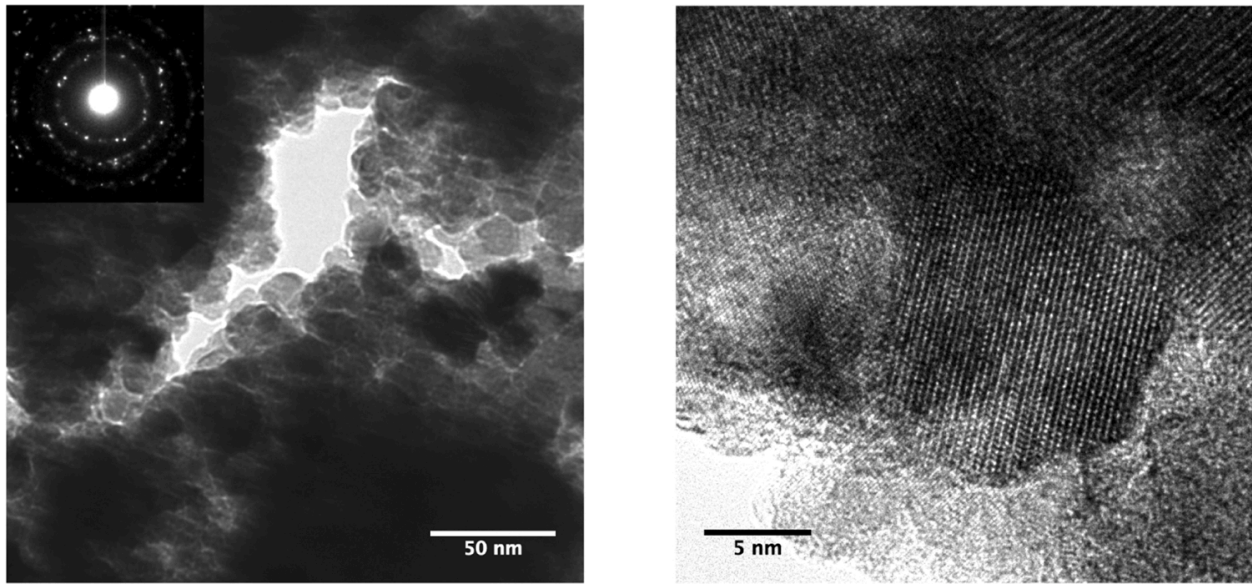

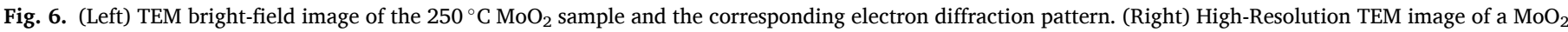
nanocrystal.

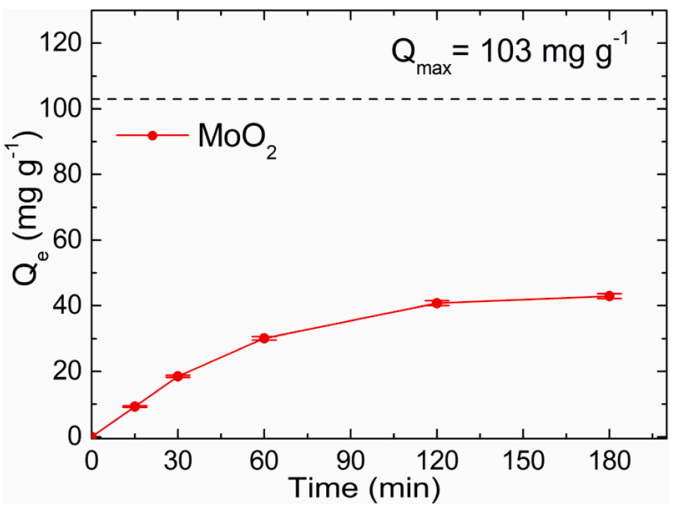

Fig. 7. $\mathrm{MB}$ adsorption capacity versus time for $\mathrm{MoO}_{2}$ (circles).

increasing the treatment temperature the type I phase increases at the expense of type II, in agreement with the supposed reduction process (OH elimination) at the base of $\mathrm{MoO}_{2}$ formation. The increasing absorption towards lower frequencies is a further confirmation of the conductive nature of the bronzes. Likewise, an increasing absorption is observed for the $\mathrm{MoO}_{2}$ spectrum.

It can be further, importantly observed that there is no evidence of bonded oleate ligands, which can be easily detected by the carboxylate stretching modes, or of other organic residuals (see also the high frequency range of the spectra in the Supporting Information). Hence the as-prepared $\mathrm{MoO}_{2}$ nanoparticles were chemically pure soon after the solvothermal treatment and the purification procedure. The reason for this result is not clear, but it is similar to the properties of $\mathrm{WO}_{3}$ nanocrystals also prepared by chloroalkoxide processing in oleic acid [27]. Fig. 6 shows representative TEM images of the $250^{\circ} \mathrm{C}$ sample. Aggregation of the nanocrystals is clearly seen, and is closely related to the lack of surface ligands, as just discussed. On the other hand, nanocrystalline structures, with a size in close agreement with the XRD determination, can be observed, all featuring the $\mathrm{MoO}_{2}$ crystal structure.

Fig. S3 in Supporting Information also shows a SEM image of the $250{ }^{\circ} \mathrm{C} \mathrm{MoO}_{2}$ sample. It can be seen that the materials are very porous and tend to form some spheroidal aggregates. The adsorption/desorption isotherm, reported in the Supporting Information (Fig. S4), shows a Type IV behavior, typical of mesoporous materials, with a mean pore size of about $11 \mathrm{~nm}$. The specific surface area of the sample, as determined by BET method, was $30.4 \mathrm{~m}^{2} / \mathrm{g}$.

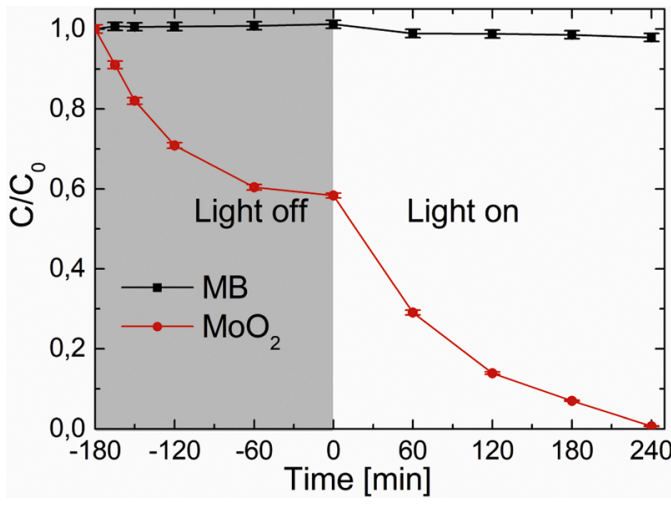

Fig. 8. Adsorption (in the dark) and photocatalytic (under solar simulator) experiment in presence of $\mathrm{MoO}_{2}$ (circles) compared to pure MB (squares).

\subsection{Adsorption and photodegradation studies}

The adsorption properties of $\mathrm{MoO}_{2}$ nanocrystals were investigated at room temperature. The sample powders were immersed in an aqueous solution of MB for three hours, until the equilibrium was reached, so to evaluate the amount of adsorbed MB. At time $t(\mathrm{~min})$ the adsorption capacity, $Q_{t}\left(\mathrm{mg} \mathrm{g}^{-1}\right)$, was calculated by the following equation [29]:

$Q_{e}=\frac{\left(C_{0}-C_{t}\right) \times V}{W}$

where $C_{0}\left(\mathrm{mg} \mathrm{L}^{-1}\right)$ is the initial concentration of $\mathrm{MB}, C_{t}\left(\mathrm{mg} \mathrm{L}^{-1}\right)$ is the concentration of $\mathrm{MB}$ at time $t, V(\mathrm{~L})$ is the solvent volume (i.e., deionized water), and $W(\mathrm{~g})$ is the mass of adsorbent used in the adsorption process. The results of this experiment are reported in Fig. 7.

After three hours the equilibrium between the $\mathrm{MoO}_{2}$ nanocrystals and MB was reached and the adsorption capacity of the nanopowders was $\sim 43 \mathrm{mg} \mathrm{g}^{-1}$. The maximum adsorption capacity $\left(Q_{\max }\right)$ was calculated by using Eq. (1) where $C_{t}$ is zero (i.e., all $\mathrm{MB}$ is adsorbed by the material). The value of $Q_{\max }$ resulted $103 \mathrm{mg} \mathrm{g}^{-1}$, and it is indicated in Fig. 7 with a dashed line. This means that $\mathrm{MoO}_{2}$ is able to remove $40 \%$ of $\mathrm{MB}$ from the solution.

When the equilibrium was reached, light was switched on in order to investigate the photodegradation properties of $\mathrm{MoO}_{2}$. This experiment was managed under a solar simulator (the evolution of the optical absorption spectra is reported in the Supporting Information, Fig. S5). Fig. 8 shows both the adsorption (in dark conditions, grey area) and the photocatalytic experiment (under solar simulator light, white area). The 

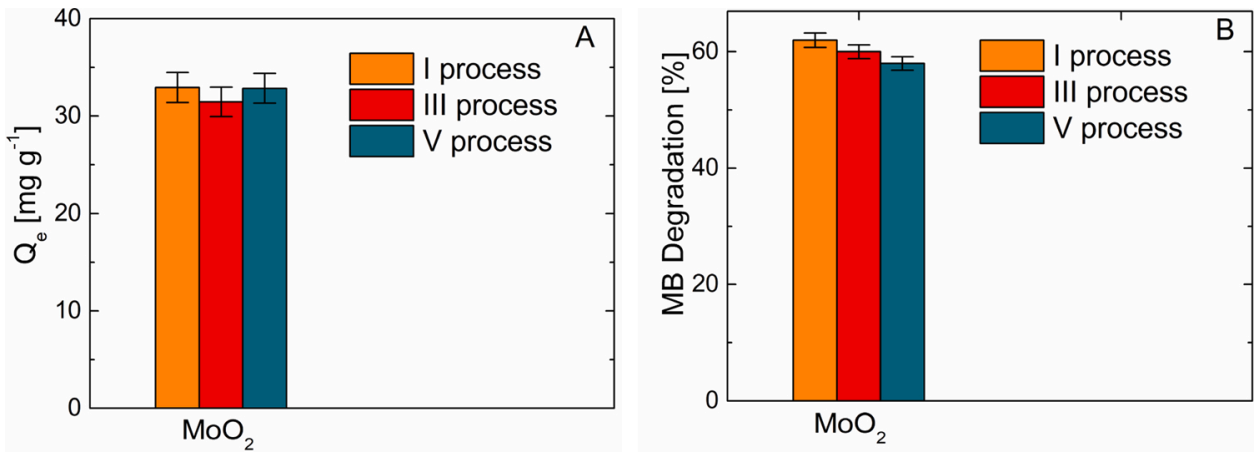

Fig. 9. Adsorption (A) and photocatalytic (B) recycling properties of $\mathrm{MoO}_{2}$ nanocrystals towards $\mathrm{MB}$.
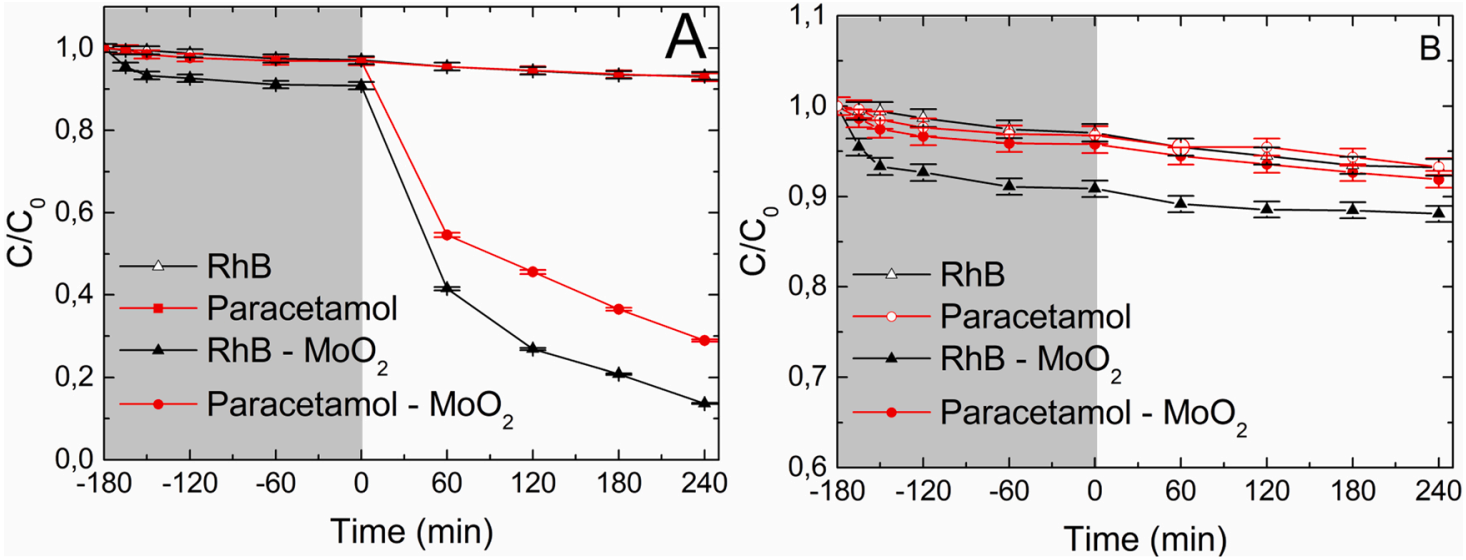

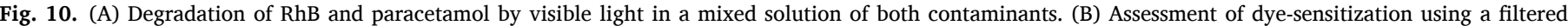
spectrum $(500-600 \mathrm{~nm}$ filtered).

data reported in the grey area have been already discussed above (Fig. 7).

$\mathrm{MoO}_{2}$ was able to degrade $\sim 60 \%$ of MB after $4 \mathrm{~h}$ of solar irradiation. Therefore by combining the adsorption and the photocatalytic activity of $\mathrm{MoO}_{2}$ nanopowders it was possible to completely remove the MB dye from the aqueous solution.

After the irradiation under visible light, the regeneration of the $\mathrm{MoO}_{2}$ nanopowders was tested by measuring the adsorption capacity for several cycles (up to 5 cycles). Fig. 9A shows the amount of adsorbed $\mathrm{MB}$, calculated by Eq. (1) at equilibrium. $\mathrm{MoO}_{2}$ removed the same amount of $\mathrm{MB}$ at least up to 5 runs of the adsorption/photocatalytic process. Similarly, the percentage of photodegraded MB is the same for all runs with $\mathrm{MoO}_{2}$ (Fig. 9B). In order to investigate the mechanism of photodegradation under visible light, the degradation of paracetamol, one of the most popular analgesic and antipyretic drugs, was investigated. Since paracetamol does not absorb in the visible region of the spectrum, any reduction in concentration can be attributed to photocatalysis onto the $\mathrm{MoO}_{2}$ support [30]. No reduction in concentration was recorded (Fig. S7). This result, in turn, suggested that the degradation of MB could be triggered by a dye sensitization process. To confirm the nature of the degradation process, measurements were performed using a mixture of RhB and paracetamol. A mixture with MB was not used

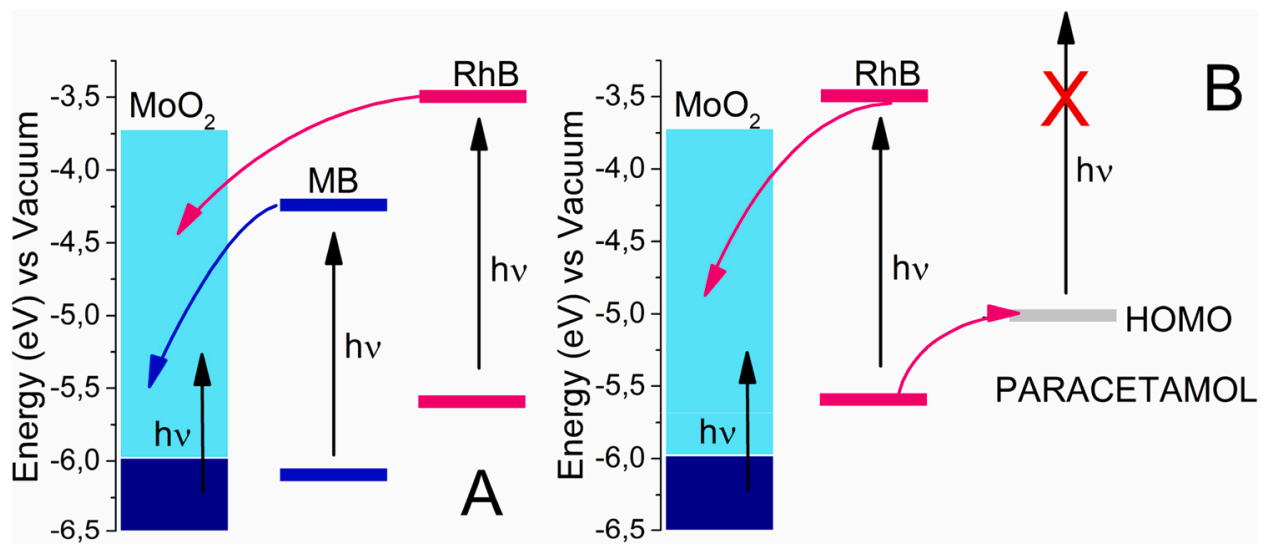

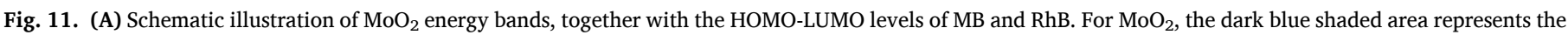

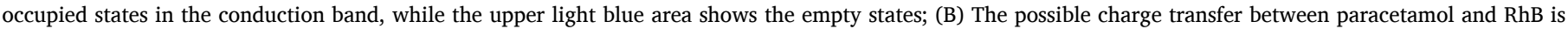
shown, circumventing the fact that paracetamol is not amenable to photoexcitation. The paracetamol LUMO is very high in energy and is not shown for clarity. 
because it has an absorption band at $246 \mathrm{~nm}$ where paracetamol absorbs, too. First of all, tests were carried out with only RhB to confirm the degradation capacity also with this dye. The results are shown in Fig. S7 and show a lower adsorption than MB but a significant photodegradation capacity: the RhB degradation was about $84 \%$ of the starting concentration after $4 \mathrm{~h}$. The degradation of the solution of paracetamol and $\mathrm{RhB}$ was then determined. The graph in Fig. 10A clearly shows the degradation of RhB and paracetamol. In particular the $\mathrm{RhB}$ degradation has a similar trend than in Fig. S7, $~ 86 \%$, and paracetamol showed a degradation of about $71 \%$. When the band absorbed by RhB (from 500 to $600 \mathrm{~nm}$ ) was filtered from the solar simulator radiation, no degradation of $\mathrm{RhB}$ or paracetamol was observed (Fig. 10B). The sequences of optical absorption spectra are reported in Fig. S8.

The mechanisms responsible for dye degradation are generally classified as direct and indirect [31,32]. In the indirect pathway, the dye can be oxidized by hydroxyl radicals triggered by holes photogenerated in a semiconducting catalyst. In the present case, however, the dye cannot count onto photogenerated holes in $\mathrm{MoO}_{2}$, since photoexcitation in the latter does not occur through the bandgap. In direct degradation mode, the dye is excited by visible light from the ground state to a triplet excited state. In such state, electron injection into the catalyst conduction band generates a dye cation and negatively charged catalyst support. The latter may give rise, as final step, to hydroxyl radicals which are responsible for final dye degradation.

As detailed in the introduction, interband absorption in $\mathrm{MoO}_{2}$ is expected for energies higher than $2.5 \mathrm{eV}$ but in the discussion of Fig. 10 it has already been noted that, upon filtering of the radiation above $500 \mathrm{~nm}$, no photodegradation was observed. These considerations exclude indirect pathways for photodegradation. On the other hand, the states above the Fermi level in $\mathrm{MoO}_{2}$ are available for accepting photoexcited electrons in the dyes, as illustrated in Fig. 11A. Data are reported for the work function of $\mathrm{MoO}_{2}$ [33] and the HOMO-LUMO values of $\mathrm{MB}$ and $\mathrm{RhB}$ [34]. For paracetamol, results from DFT modeling were used [35]. It can be seen that injection of photogenerated electrons from $\mathrm{MB}$ and $\mathrm{RhB}$ to $\mathrm{MoO}_{2}$ is favorable. This process creates the cation radicals at the base of the direct photodegradation mechanism. The overall process is made possible by the $\mathrm{MoO}_{2}$ work function, which is at lower level than the typical values for many metals [36], and of course by favorable dye adsorption onto a transition metal like Mo. In Fig. 11B, from the position of the HOMO of paracetamol (which cannot be photoexcitated, due to the very large HOMO-LUMO separation) it can be seen that hole transfer from RhB to paracetamol can occur, so creating radicals for further degradation reactions. The fact that no degradation occurred when light was not absorbed by the RhB dye (Figs. 10 and S8) nicely fits into this explanation. It is to be noted that hole transfer from RhB to paracetamol could slow down the photodegradation of RhB itself. Instead, comparison of Figs. S7 and 10 shows that $\mathrm{RhB}$ photodegradation is not perturbed by the presence of paracetamol.

Our model is necessarily approximate, due to possible fluctuations of the vacuum levels of the involved species and the fact that the HOMOLUMO levels of paracetamol should be obtained at the real working temperature. Nevertheless, such fluctuations are not expected to substantially affect the huge energy separations depicted in Fig. 11. Such kind of decomposition of synergistic pollutants has already been reported [34] but with a semiconducting support $\left(\mathrm{TiO}_{2}\right)$. In the present work, importantly, it is clarified that the metallic nature of $\mathrm{MoO}_{2}$, together with its work function, is favorable to the photodegradation promotion. One could wonder whether other materials may perform a similar function. As noted above, many metals have smaller work function than $\mathrm{MoO}_{2}$. Moreover, metals with comparable values of the work function must further display a satisfactory coordination chemistry toward the dyes, which is another advantage of a transition metal like Mo. Nevertheless, the concept of a metal photodegradation catalyst with designed work function and surface chemistry is a strong indication from the present work.

\section{Conclusions}

The solvothermal processing of Mo chloroalkoxide resulted in peculiar outcomes. From the point of view of synthetic chemistry: i) organics-free $\mathrm{MoO}_{2}$ was readily synthesized; ii) molybdenum bronzes were shown to be reducible down to $\mathrm{MoO}_{2}$; iii) for the first time it was possible to prepare molybdenum bronzes without the reduction of Mo (VI) compounds and insertion of hydrogen into the structure. From the point of view of the photodegradation properties: i) the coupling of the adsorption and photocatalytic processes resulted in complete removal of MB from the aqueous solution; ii) RhB removal was possible up to $84 \%$ of the starting concentration and, in its presence, removal of paracetamol up to $71 \%$; iii) the analysis of these results brought by the most important conclusion: the photodegradation activity cannot be explained by indirect sensitization mechanisms, simply because $\mathrm{MoO}_{2}$ is not a semiconductor, and the decisive factor was the position of the $\mathrm{MoO}_{2}$ work function. This result paves the way for potential use of metallic photodegradation catalysts by appropriate control of the work function. In the cycling experiments carried out in the present work, $\mathrm{MoO}_{2}$ did not present any stability issue. However, possible oxidation problems in long term operation should be considered in any practical implementation of the material.

\section{CRediT authorship contribution statement}

Alessandro Di Mauro: Investigation, Visualization, Writing - review \& editing. Marta Maria Natile: Investigation, Visualization, Writing review \& editing. Anton Landström: Investigation, Visualization, Writing - review \& editing. Isabella Concina: Investigation, Visualization, Writing - review \& editing. Matteo Ferroni: Investigation, Visualization, Writing - review \& editing. Vittorio Privitera: Investigation, Visualization, Writing - review \& editing. Giuliana Impellizzeri: Investigation, Visualization, Writing - review \& editing. Mauro Epifani: Conceptualization, Investigation, Visualization, Writing - original draft, Writing - review \& editing.

\section{Declaration of Competing Interest}

The authors report no declarations of interest.

\section{Acknowledgements}

This work was partially funded by the European Regional Development Funds (FESR) INTERREG V - A Italia Malta - Micro WatTS C1-1.170 (CUP: B61G18000070009). We thank Sudipto Kumar Pal and Antonio Licciulli (University of Salento) for the BET measurements. MMN acknowledges the MIUR-PON TARANTO (ARS01_00637) for partial funding. I.C. acknowledges Carl Trygger Foundation (project "Water remediation through semiconductor-supported photocatalysis - WarCat", Project No. CTS 19:70) and Kempe foundation (project: Purification platforms for the treatment of pharmaceutical wastes) for partial funding.

\section{Appendix A. Supplementary data}

Supplementary material related to this article can be found, in the online version, at doi:https://doi.org/10.1016/j.jphotochem.2021. 113258.

\section{References}

[1] B.G. Brandt, A.C. Skapski, Acta Chem. Scand. 21 (1947-1973) (1967) 661-672. [2] H.T.T. Pham, C. Jo, J. Lee, Y. Kwon, RSC Adv. 6 (2016) 17574-17582.

[3] Y.F. Zhao, Y.X. Zhang, Z.Y. Yang, Y.M. Yan, K.N. Sun, Sci. Technol. Adv. Mat. 14 (2013). 
[4] E. Zhou, C.G. Wang, Q.Q. Zhao, Z.P. Li, M.H. Shao, X.L. Deng, X.J. Liu, X.J. Xu, Ceram. Int. 42 (2016) 2198-2203.

[5] H.S. Kim, J.B. Cook, S.H. Tolbert, B. Dunn, J. Electrochem. Soc. 162 (2015) A5083-A5090.

[6] J.Y. Shi, Y. Kuwahara, M.C. Wen, M. Navlani-Garcia, K. Mori, T.C. An, H. Yamashita, Chem. - Asian J. 11 (2016) 2377-2381.

[7] Y.S. Jin, H.T. Wang, J.J. Li, X. Yue, Y.J. Han, P.K. Shen, Y. Cui, Adv. Mater. 28 (2016) 3785-3790.

[8] H. Du, X. Xie, Q. Zhu, L. Lin, Y.F. Jiang, Z.K. Yang, X. Zhou, A.W. Xu, Nanoscale 7 (2015) 5752-5759.

[9] Z.X. Yan, J.M. Xie, J.J. Jing, M.M. Zhang, W. Wei, S.B. Yin, Int. J. Hydrogen Energy 37 (2012) 15948-15955.

[10] Q.Q. Zhang, X.S. Li, Q. Ma, Q. Zhang, H. Bai, W.C. Yi, J.Y. Liu, J. Han, G.C. Xi, Nat. Commun. 8 (2017).

[11] S. Gu, M. Qin, H. Zhang, J. Ma, H. Wu, X. Qu, CrystEngComm 19 (2017) 6516-6526.

[12] J.B. Goodenough, Prog. Solid State Chem. 5 (1971) 145-399.

[13] J.B. Goodenough, Magnetism and the Chemical Bond. Interscience Monographs on Chemistry, Inorganic Chemistry Section, Interscience Division, John Wiley \& Sons, Inc., New York \& London, 1963.

[14] J.B. Goodenough, Phys. Rev. 117 (1960) 1442-1451.

[15] D.B. Rogers, R.D. Shannon, A.W. Sleight, J.L. Gillson, Inorg. Chem. 8 (1969) 841-849.

[16] T.A. Sasaki, T. Soga, H. Adachi, Phys. Status Solidi B 113 (1982) 647-655.

[17] J.K. Burdett, Inorg. Chem. 24 (1985) 2244-2253.

[18] D. Parker, J.C. Idrobo, C. Cantoni, A.S. Sefat, Phys. Rev. B 90 (2014).

[19] V. Eyert, R. Horny, K.H. Hock, S. Horn, J. Phys. Condens. Matter 12 (2000) 4923-4946.

[20] M.A.K.L. Dissanayake, L.L. Chase, Phys. Rev. B 18 (1978) 6872-6879.
[21] L.L. Chase, Phys. Rev. B 10 (1974) 2226-2231.

[22] M. Epifani, P. Imperatori, L. Mirenghi, M. Schioppa, P. Siciliano, Chem. Mater. 16 (2004) 5495-5501.

[23] L. Lutterotti, S. Matthies, H.R. Wenk, MAUD (Material Analysis Using Diffraction): A User Friendly Java Program for Rietveld Texture Analysis and More. Twelfth International Conference on Textures of Materials. (ICOTOM-12), National Research Council of Canada, Ottawa, Montreal, Canada, 1999, pp. 1599-1604.

[24] IUPAC, Compendium of chemical terminology. The "Gold Book", 2nd ed., Blackwell Scientific Publications, Oxford, 1997.

[25] N. Sotani, K. Eda, M. Sadamatu, S. Takagi, Bull. Chem. Soc. Jpn. 62 (1989) 903-907.

[26] J.J. Birtill, P.G. Dickens, Mater. Res. Bull. 13 (1978) 311-316.

[27] M. Epifani, E. Comini, R. Diaz, T. Andreu, A. Genc, J. Arbiol, P. Siciliano, G. Faglia, J.R. Morante, ACS Appl. Mater. Interfaces 6 (2014) 16808-16816.

[28] K. Eda, J. Solid State Chem. 83 (1989) 292-303.

[29] A. Bonilla-Petriciolet, D.I. Mendoza-Castillo, H.E. Reynel-Ávila (Eds.), Adsorption Processes for Water Treatment and Purification, Springer International Publishing, 2017.

[30] N. Villota, J.M. Lomas, L.M. Camarero, J. Photochem. Photobiol. A 329 (2016) $113-119$.

[31] A. Ajmal, I. Majeed, R.N. Malik, H. Idriss, M.A. Nadeem, RSC Adv. 4 (2014) 37003-37026.

[32] Y.-H. Chiu, T.-F.M. Chang, C.-Y. Chen, M. Sone, Y.-J. Hsu, Catalysts 9 (2019).

[33] M.T. Greiner, M.G. Helander, W.-M. Tang, Z.-B. Wang, J. Qiu, Z.-H. Lu, Nat. Mater. 11 (2012) 76-81.

[34] Z. Zhang, Y. Yu, P. Wang, ACS Appl. Mater. Interfaces 4 (2012) 990-996.

[35] H. Ghasempour, M. Dehestani, S.M.A. Hosseini, Struct. Chem. 31 (2020) 1403-1417, https://doi.org/10.1007/s11224-020-01499-8.

[36] G.N. Derry, M.E. Kern, E.H. Worth, J. Vac. Sci. Technol. A 33 (2015), 060801. 